\title{
Leg Space Observer on Biarticular Actuated Two-Link Manipulator for Realizing Spring Loaded Inverted Pendulum Model
}

\author{
Yasuto Kimura \\ Department of Advanced Energy \\ School of Frontier Sciences, University of Tokyo \\ 5-1-5 Kashiwanoha Kashiwa-shi, Chiba, Japan \\ Email: kimura@hori.k.u-tokyo.ac.jp
}

\author{
Sehoon Oh , Yoichi Hori \\ Department of Electrical Engineering \\ School of Engineering, University of Tokyo \\ 7-3-1 Hongo Bunkyo-ku, Tokyo, Japan \\ Email: sehoon@hori.k.u-tokyo.ac.jp \\ hori@k.u-tokyo.ac.jp
}

\begin{abstract}
This paper proposes kinematics and a control algorithm to control a two-link manipulator to simulate a spring loaded inverted pendulum (SLIP). End-effector kinematics is derived in the reference frame that is defined along the axis that connects the first joint and the end-effector. The derivation of this kinematics reveals that a biarticular actuator is suitable for this kinematics. Based on this kinematics, a disturbance observer is designed in the same reference frame. This disturbance observer removes the unnecessary inertia coupling without calculation of Jacobian matrix.
\end{abstract}

\section{INTRODUCTION}

There have been many attempts to incorporate animal features into robotics both in hardware and control design aspects, with animal muscloskeletal structures drawing particular attention.

One of the purposes of these studies is realization of robots that help or assist humans for improving welfare or replacing people for dangerous tasks, but there are many more applications. The remarkable distinction between conventional robots and animals is the presence of actuators that act upon multiple joints. Such redundant actuators have been neglected in robotics because robot engineers cannot understand the roles of these actuators are. So, the other purpose is to elucidate the role of animal's redundant structure through comparison between conventional robots and animal inspired robots, and utilize this knowledge in medical welfare.

To investigate animal structure, Kumamoto et.al. established a 6-muscle 3-pair manipulator model [1]. This model has two monoarticular muscle pairs and one biarticular muscle pair which has redundancy. Some researches focus on the solution to this redundancy such as Phase Different Control [2], $\infty-$ norm approach [3] or Linear Planning [4], and others focus on the important role of biarticular muscle between joint stiffness and force at end effector along the direction spanning first joint and end effector [5],[6]. Furthermore, Oshima et.al. verified that the gastrocnemius, which is the biarticular muscle of the lower thigh, transfers rotational energy to thrust energy for jumping upward by a simple spring and wire model [7].

On the other hand, a considerable number of studies have been conducted on locomotion. In these researches, the Spring
Loaded Inverted Pendulum (SLIP) model is one of the most simple and useful models [8]. Based on this model, many robots have been built. Raibert realized jumping and running with almost the same mechanism as the SLIP model [9], Hyon made a robot "KenKen", which has knees and ankles, and can jump and run like a dog [10]. Niiyama built an athlete robot which runs with feedforward input to pneumatic actuators located like human limbs [11].

Although numerous studies have been made on animal muscloskeletal structures and locomotion like those above, the relationship between both is still controversial. In this paper, we focus on manipulator kinematics, statics and dynamics using a monoarticular muscle pair and a biarticular muslce pair on the reference frame on the end effector. Furthermore, in order to associate the two-link manipulator with the SLIP model, inertia decoupling of the end effector using disturbance observer is proposed.

\section{Two-Link MANiPUlator With A BiarticulaR ACTUATOR}

\section{A. Basic configuration and reference frame definition}

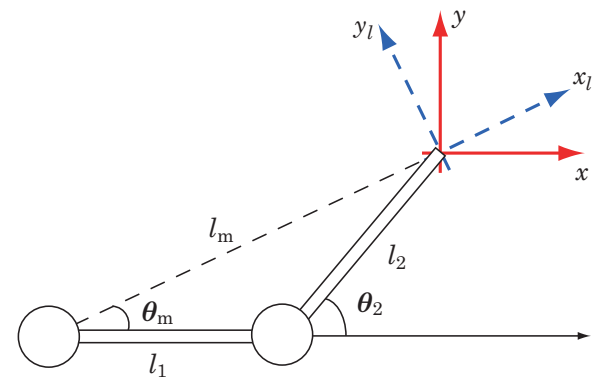

Fig. 1. Two reference frames $\Sigma$ and $\Sigma_{l}$

We have proposed kinematics in the reference frame along the first link, which is illustrated as solid lines in Figure 1. This reference frame is called the first link reference frame. Meanwhile, it is better to describe the motions of the end 
effector in the reference frame that is along the line connecting the first joint and the end effector in order to match the motions with the motions of the spring loaded inverted pendulum model. In this paper, this reference frame that is described as the dashed lines in Figure 1 is called the direct link reference frame. And, we call the coodinate space on the direct link reference frame as the "Leg Space".

\section{B. Kinematics in the direct link reference frame}

Kinematics and inverse kinematics in the first link reference frame are expressed as follows.

$$
\begin{aligned}
\left(\begin{array}{c}
\Delta x \\
\Delta y
\end{array}\right) & =\left(\begin{array}{cc}
0 & -\sin \theta_{2} \\
1 & \cos \theta_{2}
\end{array}\right)\left(\begin{array}{cc}
l_{1} & 0 \\
0 & l_{2}
\end{array}\right)\left(\begin{array}{c}
\Delta \theta_{1} \\
\Delta \theta_{12}
\end{array}\right) \\
\left(\begin{array}{c}
\Delta \theta_{1} \\
\Delta \theta_{12}
\end{array}\right) & =\frac{1}{\sin \theta_{2}}\left(\begin{array}{cc}
\frac{1}{l_{1}} & 0 \\
0 & \frac{1}{l_{2}}
\end{array}\right)\left(\begin{array}{cc}
\cos \theta_{2} & \sin \theta_{2} \\
-1 & 0
\end{array}\right)\left(\begin{array}{l}
\Delta x \\
\Delta y
\end{array}\right)
\end{aligned}
$$

New kinematics in the direct link reference frame can be derived using this kinematics and the relationship between two reference frames.

$$
\left(\begin{array}{c}
\Delta x \\
\Delta y
\end{array}\right)=\left(\begin{array}{cc}
\cos \theta_{m} & -\sin \theta_{m} \\
\sin \theta_{m} & \cos \theta_{m}
\end{array}\right)\left(\begin{array}{c}
\Delta x_{l} \\
\Delta y_{l}
\end{array}\right)
$$

where $\cos \theta_{m}=\frac{l_{1}+l_{2} \cos \theta_{2}}{l_{m}}, \sin \theta_{m}=\frac{l_{2} \sin \theta_{2}}{l_{m}}$, and $l_{m}^{2}=$ $\left(l_{1}+l_{2} \cos \theta_{2}\right)^{2}+l_{2}^{2} \sin ^{2} \theta_{2}$, by inspection of Figure 1 .

$$
\begin{aligned}
\left(\begin{array}{c}
\Delta x_{l} \\
\Delta y_{l}
\end{array}\right) & =\frac{1}{l_{m}}\left(\begin{array}{cc}
l_{1} l_{2} \sin \theta_{2} & -l_{1} l_{2} \sin \theta_{2} \\
l_{1}\left(l_{1}+l_{2} \cos \theta_{2}\right) & l_{2}\left(l_{1} \cos \theta_{2}+l_{2}\right)
\end{array}\right)\left(\begin{array}{c}
\Delta \theta_{1} \\
\Delta \theta_{12}
\end{array}\right)(4) \\
\left(\begin{array}{c}
\Delta \theta_{1} \\
\Delta \theta_{12}
\end{array}\right) & =\frac{1}{l_{m} \sin \theta_{2}}\left(\begin{array}{cc}
\cos \theta_{2}+\frac{l_{2}}{l_{1}} & \sin \theta_{2} \\
-\frac{l_{1}}{l_{2}}-\cos \theta_{2} & \sin \theta_{2}
\end{array}\right)\left(\begin{array}{c}
\Delta x \\
\Delta y
\end{array}\right)
\end{aligned}
$$

For the particular case $l_{1}=l_{2}=l$, the kinematics is simplified as follows.

$$
\begin{aligned}
\left(\begin{array}{c}
\Delta x_{l} \\
\Delta y_{l}
\end{array}\right) & =\frac{1}{l_{m}}\left(\begin{array}{cc}
l^{2} \sin \theta_{2} & -l^{2} \sin \theta_{2} \\
l^{2}\left(1+\cos \theta_{2}\right) & l^{2}\left(1+\cos \theta_{2}\right)
\end{array}\right)\left(\begin{array}{c}
\Delta \theta_{1} \\
\Delta \theta_{12}
\end{array}\right) \\
& =\frac{l^{2}}{l_{m}}\left(\begin{array}{c}
\sin \theta_{2}\left(\Delta \theta_{1}-\Delta \theta_{12}\right) \\
\left(1+\cos \theta_{2}\right)\left(\Delta \theta_{1}+\Delta \theta_{12}\right)
\end{array}\right) \\
\left(\begin{array}{c}
\Delta \theta_{1} \\
\Delta \theta_{12}
\end{array}\right) & =\frac{1}{l_{m} \sin \theta_{2}}\left(\begin{array}{cc}
\cos \theta_{2}+1 & \sin \theta_{2} \\
-\cos \theta_{2}-1 & \sin \theta_{2}
\end{array}\right)\left(\begin{array}{c}
\Delta x \\
\Delta y
\end{array}\right) \\
& =\frac{\cos \theta_{2}+1}{l_{m} \sin \theta_{2}}\left(\begin{array}{c}
1 \\
-1
\end{array}\right) \Delta x+\frac{1}{l_{m}}\left(\begin{array}{l}
1 \\
1
\end{array}\right) \Delta y
\end{aligned}
$$

The most significant point of this simplified kinematics is that the differential mode and common mode of the angles $\Delta \theta_{1}$ and $\Delta \theta_{12}$ correspond to $\Delta x_{l}$ and $\Delta y_{l}$ respectively.

\section{Statics based on the proposed Kinematics}

Statics in the direct link reference frame can be derived using the proposed kinematics. First, the statics in the first link reference frame is given as follows.

$$
\begin{aligned}
\left(\begin{array}{l}
\tau_{1}^{m} \\
\tau_{3}^{m}
\end{array}\right) & =\left(\begin{array}{cc}
l_{1} & 0 \\
0 & l_{2}
\end{array}\right)\left(\begin{array}{cc}
0 & 1 \\
-\sin \theta_{2} & \cos \theta_{2}
\end{array}\right)\left(\begin{array}{l}
f^{x} \\
f^{y}
\end{array}\right) \\
\left(\begin{array}{l}
f^{x} \\
f^{y}
\end{array}\right) & =\frac{1}{\sin \theta_{2}}\left(\begin{array}{cc}
\cos \theta_{2} & -1 \\
\sin \theta_{2} & 0
\end{array}\right)\left(\begin{array}{cc}
\frac{1}{l_{1}} & 0 \\
0 & \frac{1}{l_{2}}
\end{array}\right)\left(\begin{array}{l}
\tau_{1}^{m} \\
\tau_{3}^{m}
\end{array}\right)
\end{aligned}
$$

With the same rotation matrix, the statics in the direct link reference frame can be derived as follows.

$$
\begin{aligned}
& \left(\begin{array}{l}
\tau_{1} \\
\tau_{3}
\end{array}\right)=\frac{1}{l_{m}}\left(\begin{array}{cc}
l_{1} l_{2} \sin \theta_{2} & l_{1}\left(l_{1}+l_{2} \cos \theta_{2}\right) \\
-l_{1} l_{2} \sin \theta_{2} & l_{2}\left(l_{1} \cos \theta_{2}+l_{2}\right)
\end{array}\right)\left(\begin{array}{c}
f_{l}^{x} \\
f_{l}^{y}
\end{array}\right) \\
& \left(\begin{array}{c}
f_{l}^{x} \\
f_{l}^{y}
\end{array}\right)=\frac{1}{l_{m} \sin \theta_{2}}\left(\begin{array}{cc}
\cos \theta_{2}+\frac{l_{2}}{l_{1}} & -\frac{l_{1}}{l_{2}}-\cos \theta_{2} \\
\sin \theta_{2} & \sin \theta_{2}
\end{array}\right)\left(\begin{array}{l}
\tau_{1} \\
\tau_{3}
\end{array}\right)
\end{aligned}
$$

For the particular case $l_{1}=l_{2}=l$, the kinematics is simplified as follows.

$$
\begin{aligned}
\left(\begin{array}{c}
\tau_{1} \\
\tau_{3}
\end{array}\right) & =\frac{1}{l_{m}}\left(\begin{array}{cc}
l^{2} \sin \theta_{2} & l^{2}\left(1+\cos \theta_{2}\right) \\
-l^{2} \sin \theta_{2} & l^{2}\left(1+\cos \theta_{2}\right)
\end{array}\right)\left(\begin{array}{l}
f_{l}^{x} \\
f_{l}^{y}
\end{array}\right) \\
& =\frac{l^{2} \sin \theta_{2}}{l_{m}}\left(\begin{array}{c}
1 \\
-1
\end{array}\right) f_{l}^{x}+\frac{l^{2}\left(\cos \theta_{2}+1\right)}{l_{m}}\left(\begin{array}{l}
1 \\
1
\end{array}\right) f_{l}^{y} \\
\left(\begin{array}{c}
f_{l}^{x} \\
f_{l}^{y}
\end{array}\right) & =\frac{1}{l_{m} \sin \theta_{2}}\left(\begin{array}{cc}
\cos \theta_{2}+1 & -\cos \theta_{2}-1 \\
\sin \theta_{2} & \sin \theta_{2}
\end{array}\right)\left(\begin{array}{l}
\tau_{1} \\
\tau_{3}
\end{array}\right) \\
& =\frac{1}{l_{m} \sin \theta_{2}}\left(\begin{array}{c}
\left(1+\cos \theta_{2}\right)\left(\tau_{1}-\tau_{3}\right) \\
\sin \theta_{2}\left(\tau_{1}+\tau_{3}\right)
\end{array}\right)
\end{aligned}
$$

Force at the end effector along the $x_{l}$ and $y_{l}$ axes can also be designed by the common mode and differential mode independently; $\tau_{1}-\tau_{3}$ determines $f_{l}^{x}$ and $\tau_{1}+\tau_{3}$ determines $f_{l}^{y}$.

\section{Force SENSOR-LESS IMPEDANCE CONTROL FOR INERTIA DECOUPLING}

Even though two torques generate force at the end effector along the direct link axis, the two-link manipulator cannot work like a SLIP model, since it has a different inertia structure. To control a two-link manipulator so that it works like a SLIP model, this inertia matrix should be modified using a feedback controller. In this session, the dynamics is redescribed based on the biarticular actuator torque first, then a feedback controller is designed for modifying the inertia characteristic not only for the control torque but also for the external force.

\section{A. Inertia coupling in two-link manipulator}

Equation (14) is the conventional description of the dynamics of a two-link manipulator, where two joint torques $T_{1}^{j}, T_{2}^{j}$ are used as control input [15].

$$
\begin{aligned}
\left(\begin{array}{c}
J_{1}+ \\
J_{2}+m_{2} l_{1}^{2}+2 m_{2} l_{1} d_{2} \cos \theta_{2} \\
J_{2}+m_{2} l_{1} d_{2} \cos \theta_{2}
\end{array}\right)\left(\begin{array}{c}
m_{2} l_{1} d_{2} \cos \theta_{2} \\
J_{2}
\end{array}\right)\left(\begin{array}{c}
\ddot{\theta}_{1} \\
\ddot{\theta}_{2}
\end{array}\right) \\
\quad+\left(\begin{array}{c}
-m_{2} l_{1} d_{2} \sin \theta_{2}\left(\dot{\theta}_{2}^{2}+2 \dot{\theta}_{1} \dot{\theta}_{2}\right) \\
m_{2} l_{1} d_{2} \sin \theta_{2} \dot{\theta}_{1}^{2}
\end{array}\right) \\
\quad+\left(\begin{array}{c}
g\left(m_{1} d_{1}+m_{2} l_{1}\right) \cos \theta_{1}+g m_{2} d_{2} \cos \left(\theta_{1}+\theta_{2}\right) \\
g m_{2} d_{2} \cos \left(\theta_{1}+\theta_{2}\right)
\end{array}\right) \\
=\left(\begin{array}{c}
T_{1}^{j} \\
T_{2}^{j}
\end{array}\right)
\end{aligned}
$$

The dynamics can be simplified using the biaritcular actuator. Elements of the inertia matrix can be grouped into

$$
A_{1}=J_{1}+m_{2} l_{1}^{2}, A_{2}=J_{2}, A_{12}=m_{2} l_{1} d_{2} \cos \theta_{2} .
$$


The inertia matrix in Equation (14) can then be written as

$$
\begin{aligned}
& \left(\begin{array}{cc}
J_{1}+J_{2}+m_{2} l_{1}^{2}+2 m_{2} l_{1} d_{2} \cos \theta_{2} & J_{2}+m_{2} l_{1} d_{2} \cos \theta_{2} \\
J_{2}+m_{2} l_{1} d_{2} \cos \theta_{2} & J_{2}
\end{array}\right) \\
& =\left(\begin{array}{cc}
A_{1}+A_{2}+2 A_{12} & A_{12}+A_{2} \\
A_{12}+A_{2} & A_{2}
\end{array}\right)
\end{aligned}
$$

To express this dynamics in the coordinate of the biarticular actuator, the following conversions are used.

$$
\begin{aligned}
& \left(\begin{array}{c}
\theta_{1} \\
\theta_{12}
\end{array}\right)=\left(\begin{array}{ll}
1 & 0 \\
1 & 1
\end{array}\right)\left(\begin{array}{c}
\theta_{1} \\
\theta_{2}
\end{array}\right),\left(\begin{array}{c}
\theta_{1} \\
\theta_{2}
\end{array}\right)=\left(\begin{array}{cc}
1 & 0 \\
-1 & 1
\end{array}\right)\left(\begin{array}{c}
\theta_{1} \\
\theta_{12}
\end{array}\right) \\
& \left(\begin{array}{c}
T_{1}^{j} \\
T_{2}^{j}
\end{array}\right)=\left(\begin{array}{ll}
1 & 1 \\
0 & 1
\end{array}\right)\left(\begin{array}{c}
\tau_{1}^{m} \\
\tau_{3}^{m}
\end{array}\right),\left(\begin{array}{c}
\tau_{1}^{m} \\
\tau_{3}^{m}
\end{array}\right)=\left(\begin{array}{cc}
1 & -1 \\
0 & 1
\end{array}\right)\left(\begin{array}{c}
T_{1}^{j} \\
T_{2}^{j}
\end{array}\right)
\end{aligned}
$$

Using the above conversions, (14) can be expressed as follows.

$$
\begin{aligned}
& \left(\begin{array}{cc}
A_{1}+A_{2}+2 A_{12} & A_{12}+A_{2} \\
A_{12}+A_{2} & A_{2}
\end{array}\right)\left(\begin{array}{l}
\ddot{\theta}_{1} \\
\ddot{\theta}_{2}
\end{array}\right) \\
& +\left(\begin{array}{c}
-m_{2} l_{1} d_{2} \sin \theta_{2}\left(\dot{\theta}_{2}^{2}+2 \dot{\theta}_{1} \dot{\theta}_{2}\right) \\
m_{2} l_{1} d_{2} \sin \theta_{2} \dot{\theta}_{1}^{2}
\end{array}\right) \\
& +\left(\begin{array}{c}
g\left(m_{1} d_{1}+m_{2} l_{1}\right) \cos \theta_{1}+g m_{2} d_{2} \cos \left(\theta_{1}+\theta_{2}\right) \\
g m_{2} d_{2} \cos \left(\theta_{1}+\theta_{2}\right)
\end{array}\right) \\
& =\left(\begin{array}{l}
T_{1}^{j} \\
T_{2}^{j}
\end{array}\right) \\
& =\left(\begin{array}{cc}
A_{1}+A_{2}+2 A_{12} & A_{12}+A_{2} \\
A_{12}+A_{2} & A_{2}
\end{array}\right)\left(\begin{array}{cc}
1 & 0 \\
-1 & 1
\end{array}\right)\left(\begin{array}{c}
\ddot{\theta}_{1} \\
\ddot{\theta}_{12}
\end{array}\right) \\
& +\left(\begin{array}{c}
-m_{2} l_{1} d_{2} \sin \theta_{2}\left(\dot{\theta}_{2}^{2}+2 \dot{\theta}_{1} \dot{\theta}_{2}\right) \\
m_{2} l_{1} d_{2} \sin \theta_{2} \dot{\theta}_{1}^{2}
\end{array}\right) \\
& +\left(\begin{array}{c}
g\left(m_{1} d_{1}+m_{2} l_{1}\right) \cos \theta_{1}+g m_{2} d_{2} \cos \left(\theta_{1}+\theta_{2}\right) \\
g m_{2} d_{2} \cos \left(\theta_{1}+\theta_{2}\right)
\end{array}\right) \\
& =\left(\begin{array}{ll}
1 & 1 \\
0 & 1
\end{array}\right)\left(\begin{array}{l}
\tau_{1}^{m} \\
\tau_{3}^{m}
\end{array}\right) \\
& \left(\begin{array}{l}
\tau_{1}^{m} \\
\tau_{3}^{m}
\end{array}\right) \\
& =\left(\begin{array}{cc}
1 & -1 \\
0 & 1
\end{array}\right)\left(\begin{array}{cc}
A_{1}+A_{2}+2 A_{12} & A_{2}+A_{12} \\
A_{2}+A_{12} & A_{2}
\end{array}\right)\left(\begin{array}{cc}
1 & 0 \\
-1 & 1
\end{array}\right)\left(\begin{array}{c}
\ddot{\theta}_{1} \\
\ddot{\theta}_{12}
\end{array}\right) \\
& +\left(\begin{array}{cc}
1 & -1 \\
0 & 1
\end{array}\right)\left(\begin{array}{c}
-m_{2} l_{1} d_{2} \sin \theta_{2}\left(\dot{\theta}_{2}^{2}+2 \dot{\theta}_{1} \dot{\theta}_{2}\right) \\
m_{2} l_{1} d_{2} \sin \theta_{2} \dot{\theta}_{1}^{2}
\end{array}\right) \\
& +\left(\begin{array}{cc}
1 & -1 \\
0 & 1
\end{array}\right)\left(\begin{array}{c}
g\left(m_{1} d_{1}+m_{2} l_{1}\right) \cos \theta_{1}+g m_{2} d_{2} \cos \left(\theta_{1}+\theta_{2}\right) \\
g m_{2} d_{2} \cos \theta_{12}
\end{array}\right) \\
& =\left(\begin{array}{cc}
J_{1}+m_{2} l_{1}^{2} & m_{2} l_{1} d_{2} \cos \theta_{2} \\
m_{2} l_{1} d_{2} \cos \theta_{2} & J_{2}
\end{array}\right)\left(\begin{array}{c}
\ddot{\theta}_{1} \\
\ddot{\theta}_{12}
\end{array}\right) \\
& +\left(\begin{array}{c}
-m_{2} l_{1} d_{2} \sin \theta_{2} \dot{\theta}_{12}^{2} \\
m_{2} l_{1} d_{2} \sin \theta_{2} \dot{\theta}_{1}^{2}
\end{array}\right)+\left(\begin{array}{c}
g\left(m_{1} d_{1}+m_{2} l_{1}\right) \cos \theta_{1} \\
g m_{2} d_{2} \cos \theta_{12}
\end{array}\right)
\end{aligned}
$$

With this inertia matrix and the kinematics derived in the previous session, the operational inertia matrix in the first link reference frame is derived as follows.

$$
\begin{aligned}
\Lambda= & \left(J^{T}\right)^{-1} A J^{-1} \\
= & \frac{1}{\sin \theta_{2}}\left(\begin{array}{cc}
\cos \theta_{2} & -1 \\
\sin \theta_{2} & 0
\end{array}\right)\left(\begin{array}{cc}
\frac{1}{l_{1}} & 0 \\
0 & \frac{1}{l_{2}}
\end{array}\right) . \\
& \left(\begin{array}{cc}
J_{1}+m_{2} l_{1}^{2} & m_{2} l_{1} d_{2} \cos \theta_{2} \\
m_{2} l_{1} d_{2} \cos \theta_{2} & J_{2}
\end{array}\right) . \\
& \frac{1}{\sin \theta_{2}}\left(\begin{array}{cc}
\frac{1}{l_{1}} & 0 \\
0 & \frac{1}{l_{2}}
\end{array}\right)\left(\begin{array}{cc}
\cos \theta_{2} & \sin \theta_{2} \\
-1 & 0
\end{array}\right) \\
= & \frac{1}{\sin ^{2} \theta_{2}}\left(\begin{array}{cc}
\cos \theta_{2} & -\sin \theta_{2} \\
\sin \theta_{2} & \cos \theta_{2}
\end{array}\right)\left(\begin{array}{cc}
\frac{J_{1}}{l_{1}^{2}}+m_{2} & 0 \\
0 & 0
\end{array}\right) . \\
& \left(\begin{array}{ccc}
\cos \theta_{2} & \sin \theta_{2} \\
-\sin \theta_{2} \cos \theta_{2}
\end{array}\right)+\frac{1}{\sin ^{2} \theta_{2}}\left(\begin{array}{cc}
\frac{J_{2}}{l_{2}^{2}} & 0 \\
0 & 0
\end{array}\right) \\
& -\frac{m_{2} d_{2}}{l_{2} \sin ^{2} \theta_{2}}\left(\begin{array}{cc}
2 \cos ^{2} \theta_{2} & \cos \theta_{2} \sin \theta_{2} \\
\cos \theta_{2} \sin _{2} & 0
\end{array}\right)
\end{aligned}
$$

The inertia matrix along the direct link axis is derived using a rotation matrix based on the following relationship.

$$
\begin{gathered}
\left(\begin{array}{c}
f_{l}^{x} \\
f_{l}^{y}
\end{array}\right)=\left(\begin{array}{cc}
\cos \theta_{m} & \sin \theta_{m} \\
-\sin \theta_{m} & \cos \theta_{m}
\end{array}\right) \Lambda\left(\begin{array}{cc}
\cos \theta_{m} & -\sin \theta_{m} \\
\sin \theta_{m} & \cos \theta_{m}
\end{array}\right)\left(\begin{array}{c}
\ddot{x}_{l} \\
\ddot{y}_{l}
\end{array}\right) \\
\Lambda_{l}=\left(\begin{array}{cc}
\cos \theta_{m} & \sin \theta_{m} \\
-\sin \theta_{m} & \cos \theta_{m}
\end{array}\right) \Lambda\left(\begin{array}{cc}
\cos \theta_{m} & -\sin \theta_{m} \\
\sin \theta_{m} & \cos \theta_{m}
\end{array}\right)
\end{gathered}
$$

Since $\cos \theta_{m}=\frac{l_{1}+l_{2} \cos \theta_{2}}{l_{m}}$ and $\sin \theta_{m}=\frac{l_{2} \sin \theta_{2}}{l_{m}}$, the inertia matrix $\Lambda_{m}$ is given as

$$
\begin{aligned}
\Lambda_{l} & \left(\begin{array}{cc}
\left(l_{1} \cos \theta_{2}+l_{2}\right)^{2} & l_{1} \sin \theta_{2}\left(l_{1} \cos \theta_{2}+l_{2}\right) \\
l_{1} \sin \theta_{2}\left(l_{1} \cos \theta_{2}+l_{2}\right) & l_{1}^{2} \sin ^{2} \theta_{2}
\end{array}\right) . \\
& \frac{1}{l_{m}^{2} \sin ^{2} \theta_{2}}\left(\frac{J_{1}}{l_{1}^{2}}+m_{2}\right)+\frac{1}{l_{m}^{2} \sin ^{2} \theta_{2}} \frac{J_{2}}{l_{2}^{2}} . \\
& \left(\begin{array}{cc}
\left(l_{1}+l_{2} \cos \theta_{2}\right)^{2} & -l_{2} \sin \theta_{2}\left(l_{1}+l_{2} \cos \theta_{2}\right) \\
-l_{2} \sin \theta_{2}\left(l_{1}+l_{2} \cos \theta_{2}\right) & l_{2}^{2} \sin ^{2} \theta_{2}
\end{array}\right) \\
& -\frac{m_{2} d_{2} \cos \theta_{2}}{l_{m}^{2} l_{2} \sin ^{2} \theta_{2}} \cdot \\
& \left(\begin{array}{cc}
2\left(l_{1} \cos \theta_{2}+l_{2}\right)\left(l_{1}+l_{2} \cos \theta_{2}\right) & \sin \theta_{2}\left(l_{1}^{2}-l_{2}^{2}\right) \\
\sin \theta_{2}\left(l_{1}^{2}-l_{2}^{2}\right) & -2 l_{1} l_{2} \sin ^{2} \theta_{2}
\end{array}\right)
\end{aligned}
$$

The coupling term in this matrix is

$$
\begin{aligned}
& \frac{\left(\left(J_{1}+m_{2} l_{1}^{2}-J_{2}\right) \cos \theta_{2}+\left(\frac{J_{1}}{l_{1}^{2}}+m_{2}-\frac{J_{2}}{l_{2}^{2}}\right) l_{1} l_{2}\right)}{\sin \theta_{2} l_{m}^{2}} \\
& -\frac{m_{2} d_{2} \cos \theta_{2}}{l_{2} l_{m}^{2} \sin \theta_{2}}\left(l_{1}^{2}-l_{2}^{2}\right) .
\end{aligned}
$$

Under the condition $l_{1}=l_{2}=l$, the matrix will be

$$
\begin{aligned}
\Lambda_{l} & \left(\begin{array}{cc}
\left(\cos \theta_{2}+1\right)^{2} & \sin \theta_{2}\left(\cos \theta_{2}+1\right) \\
\sin \theta_{2}\left(\cos \theta_{2}+1\right) & \sin ^{2} \theta_{2}
\end{array}\right) . \\
& \frac{l^{2}}{l_{m}^{2} \sin ^{2} \theta_{2}}\left(\frac{J_{1}}{l_{1}^{2}}+m_{2}\right)+\frac{l^{2}}{l_{m}^{2} \sin ^{2} \theta_{2}} \frac{J_{2}}{l_{2}^{2}} .
\end{aligned}
$$




$$
\begin{gathered}
\left(\begin{array}{cc}
\left(1+\cos \theta_{2}\right)^{2} & -\sin \theta_{2}\left(1+\cos \theta_{2}\right) \\
-\sin \theta_{2}\left(1+\cos \theta_{2}\right) & \sin ^{2} \theta_{2}
\end{array}\right) \\
-\frac{m_{2} d_{2} l \cos \theta_{2}}{l_{m}^{2} \sin ^{2} \theta_{2}}\left(\begin{array}{cc}
2\left(\cos \theta_{2}+1\right)^{2} & 0 \\
0 & -2 \sin ^{2} \theta_{2}
\end{array}\right)
\end{gathered}
$$

Since $l_{m}^{2}=2 l^{2}\left(1+\cos \theta_{2}\right)$, this matrix has the following coupling term.

$$
-\frac{1}{2 \sin \theta_{2}}\left(\frac{J_{1}}{l^{2}}+m_{2}-\frac{J_{2}}{l^{2}}\right)
$$

This is different from the actual SLIP dynamics where the motions in the $x$ and $y$ directions are independent. A feedback controller can modify this inertia matrix in order to achieve independent motion.

The entire dynamics including terms representing Coriolis force and gravity can also be converted into direct link coordinates.

$$
\begin{aligned}
& \left(\begin{array}{c}
f_{l}^{x} \\
f_{l}^{y}
\end{array}\right)=\Lambda_{l}\left(\begin{array}{c}
\ddot{x}_{l} \\
\ddot{y}_{l}
\end{array}\right) \\
& +\frac{g}{l_{m} \sin \theta_{2}}\left(\begin{array}{c}
\left(1+\cos \theta_{2}\right)\left(\frac{m_{1} d_{1}+m_{2} l_{1}}{l_{1}} \cos \theta_{1}-\frac{m_{2} d_{2}}{l_{2}} \cos \theta_{12}\right) \\
\sin \theta_{2}\left(\frac{m_{1} d_{1}+m_{2} l_{1}}{l_{1}} \cos \theta_{1}+\frac{m_{2} d_{2}}{l_{2}} \cos \theta_{12}\right)
\end{array}\right) \\
& \quad-\frac{1}{l_{m}}\left(\begin{array}{c}
\left(1+\cos \theta_{2}\right)\left(\frac{\dot{\theta}_{12}^{2}}{l_{1}}+\frac{\dot{\theta}_{1}^{2}}{l_{2}}\right) \\
\sin \theta_{2}\left(\frac{\dot{\theta}_{12}^{2}}{l_{1}}-\frac{\dot{\theta}_{1}^{2}}{l_{2}}\right)
\end{array}\right)
\end{aligned}
$$

\section{B. Design of direct link space disturbance observer}

Decoupling has been achieved by a number of feedback controllers. However, it mostly acts on the inertia matrix for the control input. The most challenging point of the control design here is that the inertia matrix against the external forces should be decoupled too. We have proposed a force sensorless impedance control, where the external force is estimated using the plant dynamics, and the response to the external force can be designed based on the estimated force value. The same technology can be adopted for decoupling of the inertia matrix against the external force.

Firstly, the disturbance observer is designed in the direct link reference frame.

$$
\begin{aligned}
\left(\begin{array}{c}
\hat{d}_{l}^{x} \\
\hat{d}_{l}^{y}
\end{array}\right)= & \frac{1}{\tau_{q} s+1}\left(\left(\begin{array}{c}
f_{l}^{x} \\
f_{l}^{y}
\end{array}\right)-\Lambda_{l}\left(\begin{array}{c}
\ddot{x}_{l} \\
\ddot{y}_{l}
\end{array}\right)\right) \\
= & \frac{Q(s)}{l_{m} \sin \theta_{2}}\left(\begin{array}{c}
\left(1+\cos \theta_{2}\right)\left(\tau_{1}-\tau_{3}\right) \\
\sin \theta_{2}\left(\tau_{1}+\tau_{3}\right)
\end{array}\right) \\
& -Q(s) \Lambda_{l n} \frac{l^{2}}{l_{m}}\left(\begin{array}{c}
\sin \theta_{2}\left(\ddot{\theta}_{1}-\ddot{\theta}_{12}\right) \\
\left(1+\cos \theta_{2}\right)\left(\ddot{\theta}_{1}+\ddot{\theta}_{12}\right)
\end{array}\right)
\end{aligned}
$$

where $Q(s)$ is a low pass filter with a time constant $\tau_{q}(Q(s)=$ $\frac{1}{\tau_{q} s+1}$ ). If $\Lambda_{l n}$ is set as follows and the estimated disturbance is fed back to reject the actual disturbance, the inertia can be decoupled.

$$
\begin{aligned}
\Lambda_{l n} & =\left(\begin{array}{cc}
\Lambda_{l_{11_{n}}} & 0 \\
0 & \Lambda_{l_{22_{n}}}
\end{array}\right) \\
\Lambda_{l_{11_{n}}} & =\frac{\left(1+\cos \theta_{2}\right)^{2}}{l_{m}^{2} \sin ^{2} \theta_{2}}\left(J_{1}+m_{2} l^{2}+J_{2}-2 m_{2} d_{2} l \cos \theta_{2}\right) \\
& =\frac{1+\cos \theta_{2}}{2 l^{2} \sin ^{2} \theta_{2}}\left(J_{1}+m_{2} l^{2}+J_{2}-2 m_{2} d_{2} l \cos \theta_{2}\right) \\
\Lambda_{l_{22_{n}}} & =\frac{1}{l_{m}^{2}}\left(J_{1}+m_{2} l^{2}+J_{2}+2 m_{2} d_{2} l \cos \theta_{2}\right) \\
& =\frac{\left(J_{1}+m_{2} l^{2}+J_{2}+2 m_{2} d_{2} l \cos \theta_{2}\right)}{2 l^{2}\left(1+\cos \theta_{2}\right)}
\end{aligned}
$$

As a result, the estimated disturbance has the following expression.

$$
\begin{aligned}
\hat{d}_{l}^{x}= & \frac{1+\cos \theta_{2}}{l l_{m} \sin \theta_{2}}\left(\left(m_{1} d_{1}+m_{2} l_{1}\right) \cos \theta_{1}-m_{2} d_{2} \cos \theta_{12}\right) g \\
& -\frac{1+\cos \theta_{2}}{l l_{m}}\left(\dot{\theta}_{12}^{2}+\dot{\theta}_{2}^{2}\right)+\frac{\frac{J_{1}}{l^{2}}+m_{2}-\frac{J_{2}}{l^{2}}}{2 \sin \theta_{2}} \ddot{y}_{l}+f_{e}^{x} \\
\hat{d}_{l}^{y}= & \frac{1}{l l_{m}}\left(\left(m_{1} d_{1}+m_{2} l_{1}\right) \cos \theta_{1}+m_{2} d_{2} \cos \theta_{12}\right) g \\
& -\frac{\sin \theta_{2}}{l l_{m}}\left(\dot{\theta}_{12}^{2}-\dot{\theta}_{2}^{2}\right)+\frac{\frac{J_{1}}{l^{2}}+m_{2}-\frac{J_{2}}{l^{2}}}{2 \sin \theta_{2}} \ddot{x}_{l}+f_{e}^{y}
\end{aligned}
$$

The estimated disturbance can be removed by feedback as follows.

$$
\left(\begin{array}{c}
\tau_{1}^{f b} \\
\tau_{3}^{f b}
\end{array}\right)=\frac{l^{2}}{l_{m}}\left(\begin{array}{cc}
\sin \theta_{2} & \left(1+\cos \theta_{2}\right) \\
-\sin \theta_{2} & \left(1+\cos \theta_{2}\right)
\end{array}\right)\left(\begin{array}{c}
\hat{d}_{l}^{x} \\
\hat{d}_{l}^{y}
\end{array}\right)
$$

However, this decoupling also applies to still on the control torque $\tau_{1}, \tau_{3}$, and the external force $\left(f_{e}^{x}, f_{e}^{y}\right)$ will also be rejected.

\section{Simplification of the time varying gain to remove singular points}

Even though the Jacobian matrix is simplified to two gains, these gains still require information of $\theta_{2}$, and contain singular points where the gain becomes infinity due to division by $\sin \theta_{2}$. These time varying gains can be removed, and the disturbance observer structure can take a simpler form.

Figure 2 (a) is the block diagram of the disturbance observer designed in Equations (36) and (37), where $J_{1}^{a}=$ $\frac{J_{1}+m_{2} l^{2}+J_{2}-2 m_{2} d_{2} l \cos \theta_{2}}{2}$ and $J_{2}^{a}=\frac{J_{1}+m_{2} l^{2}+J_{2}+2 m_{2} d_{2} l \cos \theta_{2}}{2}$. $H_{1}$ and $H_{2}$ are the coefficients in Equations (36) and (37). Since the inertia $\Lambda_{1 N}$ is defined by Equations (33) to (35), the coefficients are described as follows.

$$
H_{1}=\frac{1+\cos \theta_{2}}{l_{m} \sin \theta_{2}}, H_{2}=\frac{1}{l_{m}}
$$

In order to convert the disturbance estimated in the direct link coordinates to the actual actuator coordinates, the conversion in Equation (12) should be applied to this estimation as follows. 


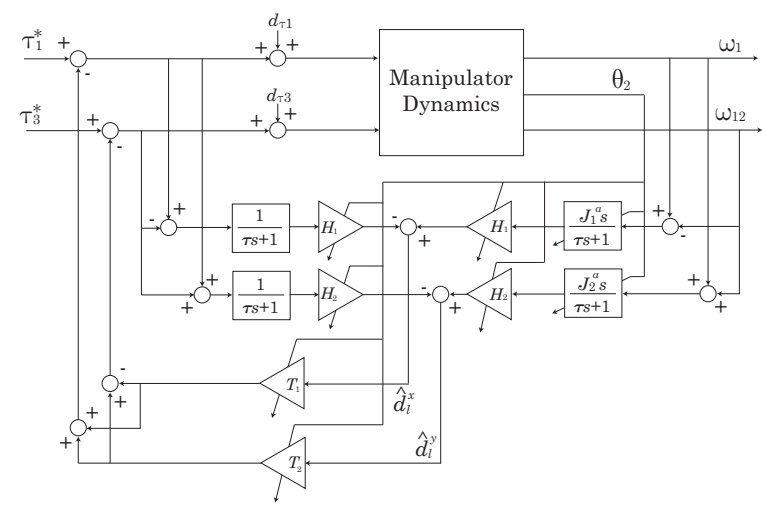

(a) Disturbance observer with variable gains

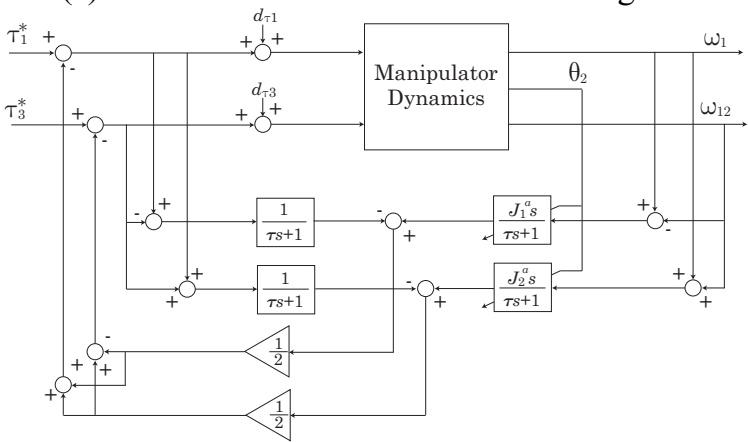

(b) Disturbance observer with constant gains

Fig. 2. Disturbance observer in the direct link axis

$$
\begin{aligned}
\left(\begin{array}{c}
\tau_{1}^{d o b} \\
\tau_{3}^{d o b}
\end{array}\right) & =\frac{l^{2} \sin \theta_{2}}{l_{m}}\left(\begin{array}{c}
1 \\
-1
\end{array}\right) \hat{d}_{l}^{x}+\frac{l^{2}\left(\cos \theta_{2}+1\right)}{l_{m}}\left(\begin{array}{l}
1 \\
1
\end{array}\right) \hat{d}_{l}^{y} \\
& =\left(\begin{array}{c}
1 \\
-1
\end{array}\right) T_{1} \hat{d}_{l}^{x}+\left(\begin{array}{l}
1 \\
1
\end{array}\right) T_{2} \hat{d}_{l}^{y},
\end{aligned}
$$

where $T_{1}=\frac{l^{2} \sin \theta_{2}}{l_{m}}, T_{2}=\frac{l^{2}\left(\cos \theta_{2}+1\right)}{l_{m}}$ correspond to $T_{1}$ and $T_{2}$ in Figure 2. As described in Figure 2, $H_{1}$ is multiplied by $T_{1}$ and $H_{2}$ is multiplied by $T_{2}$, and with the relationship $l_{m}^{2}=2 l^{2}\left(1+\cos \theta_{2}\right)$ this multiplication results in constants given in the following equations.

$$
\begin{aligned}
& H_{1} T_{1}=\frac{1+\cos \theta_{2}}{l_{m} \sin \theta_{2}} \frac{l^{2} \sin \theta_{2}}{l_{m}}=\frac{1}{2} \\
& H_{2} T_{2}=\frac{1}{l_{m}} \frac{l^{2}\left(\cos \theta_{2}+1\right)}{l_{m}}=\frac{1}{2}
\end{aligned}
$$

Figure 2 (b) is the disturbance observer redesigned after the gain multiplication where all the variable gains in Figure 2 are removed and only constant gains remain. This is a significant improvement for the disturbance observer design in a two-link manipulator. All the conversions from the operational space (which is defined as the direct link coordinates) to the actuator space are conducted using constant gains; there is no need to use the information of $\theta_{2}$ and there is no division by $\sin \theta_{2}$. Even though we have state dependent variables $J_{1}^{\text {all }}, J_{2}^{\text {all }}$, the independent term is $\cos \theta_{2}$ and there is no singular point where the conversion becomes impossible. Using the proposed
TABLE I

PARAMETER OF SiMULATION

\begin{tabular}{|c|c|c|c|}
\hline$m_{1}$ & $1.0 \mathrm{~kg}$ & $m_{2}$ & $1.0 \mathrm{~kg}$ \\
\hline$l_{1}$ & $0.25 \mathrm{~m}$ & $l_{2}$ & $0.25 \mathrm{~m}$ \\
\hline$d_{1}$ & $0.125 \mathrm{~m}$ & $d_{2}$ & $0.125 \mathrm{~m}$ \\
\hline$J_{1}$ & $0.0208 \mathrm{~kg} \cdot \mathrm{m}^{2}$ & $J_{2}$ & $0.0208 \mathrm{~kg} \cdot \mathrm{m}^{2}$ \\
\hline$\theta_{1}$ & $\pi / 4$ & $\theta_{2}$ & $-\pi / 2$ \\
\hline
\end{tabular}

disturbance observer, disturbances are rejected regardless of the posture of the manipulator at the singular point where it had been difficult to control the manipulator.

\section{Estimation of external force}

The estimated disturbance is lumped information given by Equation (36) and (37), which includes inertial coupling, Coriolis force and external force. In order to obtain the external force information, other disturbances should be removed. Since the Coriolis force is comparatively small, we will focus only the removal of the inertia coupling.

As the coupling inertia is given in a simple form, this inertia value can be used to remove the coupling disturbance and the external force estimation can be given as follows.

$$
\begin{aligned}
& \hat{f}_{x}=\hat{d}_{x}-\frac{J_{1}+m_{2} l^{2}-J_{2}}{2 l^{2} \sin \theta_{2}} \frac{l^{2}\left(1+\cos \theta_{2}\right)}{l_{m}} Q(s)\left(\ddot{\theta}_{1}+\ddot{\theta}_{12}\right) \\
& \hat{f}_{y}=\hat{d}_{y}-\frac{J_{1}+m_{2} l^{2}-J_{2}}{2 l^{2} \sin \theta_{2}} \frac{l^{2} \sin \theta_{2}}{l_{m}} Q(s)\left(\ddot{\theta}_{1}-\ddot{\theta}_{12}\right)
\end{aligned}
$$

\section{E. Simulation Results}

Here, we show some simulation results of the Leg Space Observer(LSO) for a planar two-link manipulator. We used Open Dynamics Engine for 3D graphical simulation, and Table.I lists the parameters used.

The results of velocity control with and without the LSO are described in Figure 3. In Figure 3, $\dot{x}_{l}=0.5 \sin (2 \pi 2 t), \dot{y}_{l}=$ 0 . It is obvious that the LSO can reject the effect of inertia coupling, and $\dot{x_{l}}$ tracks its reference.

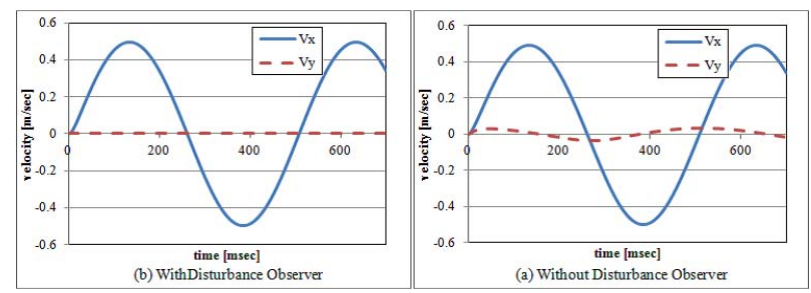

Fig. 3. Velocity Reference of $2 \mathrm{~Hz}$ sinusoidal wave for $\dot{x_{l}}$

Figure 4 is the result of velocity control using simplified LSO described in Figure 2(b), and the reference is $1.0[\mathrm{~Hz}]$ sinusoidal wave on only $x_{l}$ and the disturbance is cosine wave on $y_{l}$. Despite only using constant gains, the LSO can reject disturbances.

If we set nominal inertia matrix as (33), the inertia coupling between the $x_{l}$ and $y_{l}$ components are canceled, and the twolink leg can behave like a linear actuator which moves along 


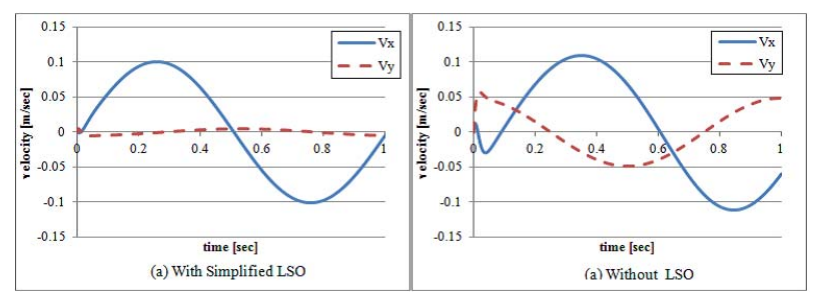

Fig. 4. Performance of simplified Simplified LSO

only the $x_{l}$ axis when external forces act on that axis. Figure 5 describes the end effector velocity along the $x_{l}$ and $y_{l}$ axes in which the case with only disturbance rejection on $y_{l}$ axis and the case without any disturbance rejection. In (a), the end effector moves only in the $x_{l}$ direction, with high stiffness in the $y_{l}$ direction. On the other hand, in (b), the end effector moves on not only in the $x_{l}$ direction but also in the $y_{l}$ direction because of the inertia coupling.
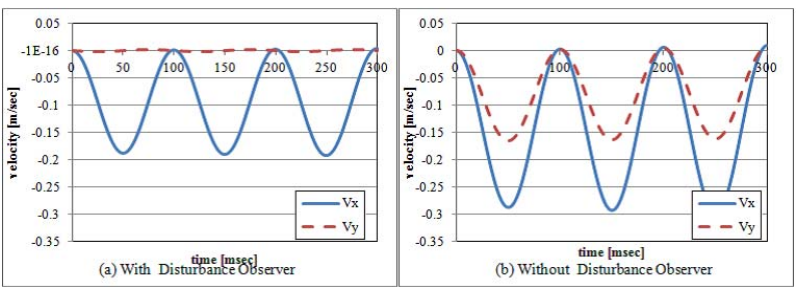

Fig. 5. Reaction to external force along $x_{l}$-axis

\section{CONCLUSION}

We suggested new methods for calculation kinematics, statics and dynamics for a two-link manipulator equipped with one biarticuar actuator and one monoarticular actuator on the direct link reference frame. In this new leg space dynamics, the inertia matrix can be described simply, clearly showing that the inertia matrix has coupling terms. Therefore, the leg space observer (LSO) can be designed in simple form without using a Jacobian matrix, and its effectiveness for removing inertia coupling is verified by simulations.

To make legged robots that vehave according to the SLIP model, we will design force sensorless impedance control using algorithms of FSPAC in [14]. Figure 6 shows the leg space observer based impedance controller (LeSOIC). With the inner disturbance observer, the inertia coupling including Coriolis force, gravity and the external force are removed. The outer disturbance observer estimates the external force again, and the desired impedance $\left(P_{M}^{x}, P_{M}^{y}\right)$ against this external force is designed. The output of the plant will track the reference generated by the desired impedance due to the feedback controller $\left(C^{x}\right.$ and $\left.C^{y}\right)$. With this outer disturbance observer and the feedback controller, the external forces will not be removed and the response to the forces can be controlled.

Furthermore, we are developing an articulated hopping robot which uses a biarticular muscle as a mechanical spring, and we plan to verify the effectiveness of LeSOIC.

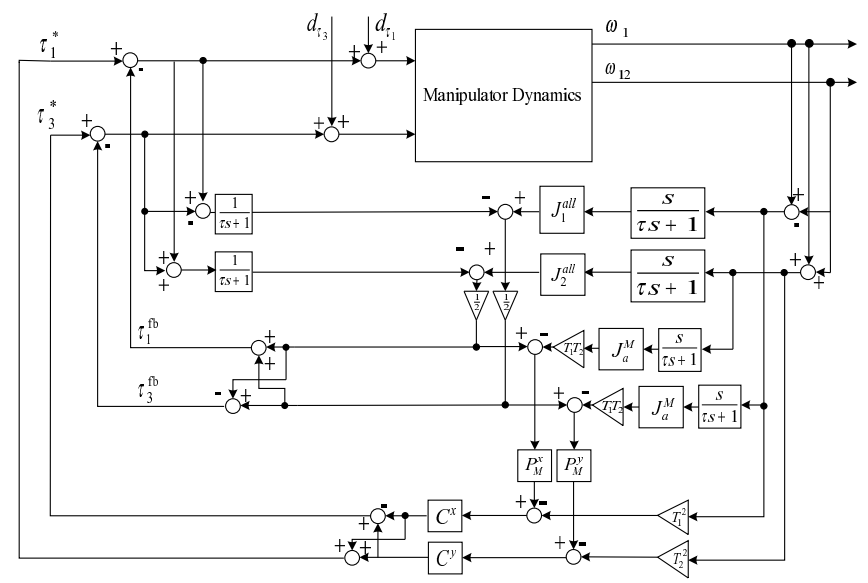

Fig. 6. Force sensor-less impedance control

\section{REFERENCES}

[1] Minayori Kumamoto, Toru Oshima, Tomohisa Yamamoto, "Control properties induced by existence of antagonistic pairs of bi-articular muscles -Mechanical engineering model analyses," Human Movement Science, vol. 13, pp. 611-634, 1994

[2] T. Tsuji, "A model of antagonistic triarticular muscle mechanism for lancelet robot," Proc. 11th IEEE Int. Workshop on Advanced Motion Control, 2010, pp. 496-501

[3] V. Salvucci, S. Oh, and Y. Hori, "Infnity norm approach for precise force control of manipulators driven by bi-articular actuators," Proc. 36th Annu. Conf. IEEE Ind. Electron, 2010, pp. 1908-1913

[4] T. Sugimoto, H. Fukusho, and T. Koseki, "A proposal of maximizing method of output force and acceleration for a manipulator with kinematic and muscular redundancy using linear programming method and its engineering verication," Journal of the Robotics Society of Japan, 2011 [submitted]

[5] S. Oh, Y. Kimura, Y. Hori, "Reaction Force Control of Robot Manipulator Based on Biarticular Muscle Viscoelasticity Control," Proc. AIM, 2010, pp.1105 - 1110.

[6] Y. Kimura, S. Oh, and Y. Hori, "Novel reaction force control design based on bi-articular driving system using intrinsic muscle viscoelasticity," Proc. IEEE International Conference on Mechatronics, 2011, pp. 815-820.

[7] T. Oshima, K. Toriumi and T. Fujikawa and N. Momose, "Jump mechanism using coordination function of bi-articular muscle in knee and ankle joint (in Japanese)," Journal of the Japan Society of Mechanical Engineer, C, vol. 71, No. 712, pp. 176-182, 2005.

[8] R. Blickhan, "The spring-mass model for running and hopping," Journal of Biomechanics, vol. 22, no. 11-12, pp. 1217-1227, 1989.

[9] M. H. Raibert, "Legged robots," Communications of the ACM, vol. 29, no. 6, pp. 499-514, May. 1986.

[10] S. H. Hyon and T. Mita, "Development of a biologically inspired hopping robot-eKenkenf," Proc. IEEE International Conference on Robotics and Automation (Cat. No.02CH37292), vol. 4, no. May, pp. 3984-3991, 2002.

[11] R. Niiyama, S. Nishikawa, and Y. Kuniyoshi, "Musculoskeletal force control for bipedal running of an athlete robot,"Proc. Annu. Conf. of the Robotics Society of Japan, 2010, pp. 7-10.

[12] N. Hogan, "Adaptive control of mechanical impedance by coactivation of antagonist muscles," IEEE Transactions on Automatic Control, vol. 29, no. 8, pp. 681-690, Aug. 1984

[13] S. Oh, V. Salvucci, Y. Kimura, Y. Hori, "Verification of effcient Force transmission by biarticular muscle actuator," Proc. International Federation of Automatic Control, pp 13516-13521, 2011

[14] S. Oh and Y. Hori, "Generalized discussion on design of force-sensorless power assist control," IEEE International Workshop on Advanced Motion Control, pp.492-497, 2008

[15] S. Oh and Y. Hori, "Development of two-degree-of-freedom control for robot manipulator with biarticular muscle torque," American Control Conference, 2009. ACC, pp. 325-330, 2009. 